\title{
The Impact of Precision Uncertainty on Predictive Accuracy Metrics of Non-Animal Testing Methods
}

\author{
Maria Leontaridou 1,2,3, Silke Gabbert ${ }^{1}$ and Robert Landsiedel 3 \\ ${ }^{1}$ Wageningen University, Environmental Economics and Natural Resources Management Group, Wageningen, The Netherlands; ${ }^{2}$ Current address: \\ Triskelion B.V., Zeist, The Netherlands; ${ }^{3}$ BASF SE, Experimental Toxicology and Ecology, Ludwigshafen, Germany
}

\begin{abstract}
The ability of non-animal methods to correctly predict the outcome of in vivo testing in repeated applications is referred to as precision. Due to dichotomizing continuous read-outs into discrete "positive/negative" hazard data, non-animal methods can reveal discordant classifications if results are sufficiently close to a defined classification threshold. This paper explores the impact of precision uncertainty on the predictive accuracy of non-animal methods. Using selected non-animal methods for assessing skin sensitization hazard as case study examples, we explore the impact of precision uncertainty separately and in combination with uncertainty due to varying composition and size of experimental samples. Our results underline that discrete numbers on a non-animal method's sensitivity, specificity, and concordance are of limited value for evaluation of its predictivity. Instead, information on the variability and the upper and lower limits of accuracy metrics should be provided to ensure a transparent assessment of a testing method's predictivity, and to allow for a meaningful comparison of the predictivity of a non-animal method with that of an animal test.
\end{abstract}

\section{Introduction}

Although animal studies do not accurately predict human toxicity (Russell and Burch, 1959), they are still regarded as the "gold standard" and results of so-called "alternative methods" (also termed "non-animal methods" or "new approach methods") are compared to results of respective animal studies. In rare cases, sufficient data from humans are available to serve as a reference for comparison. The degree of agreement between results obtained by in vivo and non-animal methods is quantified as accuracy metrics. Several studies have pointed to possible biases in non-animal testing methods' accuracy metrics due to inter- and intra-laboratory variability, which can impact their reliability (Weil and Scala, 1971; Agnese et al., 1984; Margolin et al., 1984; Worth and Cronin, 2001a; Hothorn, 2002, 2003). The analysis of inter-laboratory variability, i.e., the reproducibility of test results across different, independent laboratories, has received wide attention in the scientific literature (Sakaguchi et al., 2010; Sirota et al., 2014). Though the relevance of intra-laboratory variability, i.e., the ability of in vivo tests or non-animal methods to reproduce outcomes in repeated experimental applications, has been recognized (Bruner et al., 1996), systematic analyses of intra-laboratory variability have not been taken up for a long time.
Just recently, several studies assessing the intra-laboratory reproducibility of testing methods used for skin sensitization hazard classification became available. For the gold standard animal test, i.e., the local lymph node assay (LLNA), Dumont et al. (2016), Hoffmann (2015), and Kolle et al. (2013) analyzed the variability of classifications caused by dichotomization of continuous readouts into discrete "positive/negative" data. In particular, Kolle et al. (2013) determined a range around the classification threshold within which the LLNA reveals discordant results in repeated applications. This range has been called the "grey zone" (Dimitrov et al., 2016) or "borderline range" (BR) (Kolle et al., 2013). The studies concluded that substances yielding test results within the BR can be classified as neither positive nor negative, but must be reported as "inconclusive" or "ambiguous". This limits the LLNA's precision, i.e., its ability to reveal concordant results in repeated applications.

We previously quantified the BR for the LLNA (Leontaridou et al., 2017) and selected non-animal testing methods, i.e., the Direct Peptide Reactivity Assay (DPRA) (Gerberick et al., 2004, 2007), the ARE-Nrf2 luciferase method LuSens (Ramirez et al., 2014, 2016), and the human cell line activation test (h-CLAT) (Ashikaga et al., 2006, 2010; Sakaguchi et al., 2006, 2010). Furthermore, we determined borderline substances in the experimen-
Received October 11, 2018: Accepted March 27, 2019; Epub April 5, 2019; ( The Authors, 2019.

ALTEX 36(3), 435-446. doi:10.14573/altex.1810111

Correspondence: Robert Landsiedel, PhD

BASF Experimental Toxicology and Ecology

BASF SE, GB/TB - Z470, 67056 Ludwigshafen, Germany

(robert.landsiedel@basf.com)
This is an Open Access article distributed under the terms of the Creative Commons Attribution 4.0 International license (http://creativecommons.org/licenses/by/4.0/) which permits unrestricted use, distribution and reproduction in any medium, provided the original work is appropriately cited. 
tal sample tested with the " 2 out of 3 " integrated testing strategy (ITS), which is a combination of the aforementioned non-animal testing methods (Bauch et al., 2012; Urbisch et al., 2015). Our analysis showed that the number and percentage of substances considered borderline can be significant and depend on the relationship between the size of the BR and the substances yielding results within this range.

Clearly, substances with ambiguous hazard classification cannot contribute to determining a testing method's predictive accuracy. Consequently, ignoring the BR in a testing method's prediction model - and, hence, precision uncertainty - may bias the assessment of classification accuracy. So far, however, the size and direction of this bias has not been explored systematically.

The aim of this paper is, therefore, to examine the impact of precision uncertainty on sensitivity, specificity, and concordance. For our analysis we used non-animal testing methods for skin sensitization hazard classification, i.e., the DPRA, LuSens, and the h-CLAT, as case study examples. Since standalone non-animal testing methods are considered to provide insufficient information for hazard classification (ECHA, 2016; Mehling et al., 2012; Reisinger et al., 2015), we also included the "2 out of 3" ITS in the analysis. Skin sensitization hazard seems to be a particularly suitable endpoint because it is required for safety evaluations of new and existing substances in different regulatory frameworks of the European Union (e.g., the REACH legislation (EC, 2006), the Cosmetics regulation (EC, 2009)). Furthermore, both human and animal data are available as a reference and several non-animal methods and testing strategies are already in regulatory use (Kleinstreuer et al., 2018; Sauer et al., 2016). Accuracy metrics are determined in relation to the LLNA and to human reference data. We analyze the impact of precision uncertainty on accuracy metrics of the selected non-animal methods, disregarding the limited precision of the in vivo test. While this may be a simplification, it reflects the postulates underlying the validation process of non-animal methods.

For our analysis we adopted a step-wise approach: First, we compared sensitivity, specificity, and concordance derived from experimental samples including borderline substances (in the following called "the full sample") with accuracy values obtained after borderline substances were excluded (in the following called "the adapted sample"). Besides the specification of the classification threshold, a testing method's accuracy depends on the size and composition of the experimental set of substances. This can vary considerably across testing methods, depending on the availability of robust in vivo and, if applicable, human data, a balanced representation of sensitization potency classes, and a balanced number of sensitizers and non-sensitizers (ECVAM, 2012, 2013). Furthermore, there is no defined minimum number of substances below which an experimental sample would be considered insufficient for robust evaluations of a non-animal testing method's predictive accuracy. Recently, the OECD defined performance standards for the ARE-Nrf2 luciferase method, recommending that a minimum list of 20 reference chemicals should be used for assessing the testing method's predictive capacity and reproducibility (OECD, 2015b; Kolle et al., 2019).

Therefore, second, we examined the impact of precision uncertainty on a non-animal testing method's accuracy in combi- nation with uncertainty due to variations of sample size. Using non-parametric bootstrapping analysis (Wehrens et al., 2000), we created randomized experimental samples for every non-animal testing method considered. This generated sample distributions of sensitivity, specificity, and concordance for each experimental sample. We quantified the mean, the standard deviation, and the $95 \%$ confidence limits for all accuracy metrics and compared mean accuracy metrics to those obtained from the sample of substances used in validation studies. Moreover, we examined the joint impact of precision uncertainty and varying sample composition by comparing accuracy metrics from randomized full samples (i.e., including borderline substances) with those retrieved from adapted samples (i.e., excluding borderline substances). Third, we analyzed the joint impacts of sample composition, sample size, and precision uncertainty on classification accuracy of the selected non-animal methods and the ITS.

\section{Materials and methods}

\subsection{Non-animal testing methods}

Responding to the urgent need to minimize animal testing, several non-animal testing methods and integrated testing strategies for assessing skin sensitization hazard have been developed (Mehling et al., 2012; Reisinger et al., 2015; Urbisch et al., 2015). The methods used for our analysis are briefly described below.

\section{The Direct Peptide Reactivity Assay (DPRA)}

The DPRA (Gerberick et al., 2004, 2007) measures the depletion of two model peptides containing a cysteine or lysine residue as a reactive nucleophilic center after incubation with a test substance. If the mean cysteine and lysine peptide depletion is above $6.38 \%$, when compared to depletion in the reference control, the test result is positive and the substance is considered to be peptide-reactive. The DPRA was validated by the European Centre for Validation of Alternative Methods (ECVAM) (EURL ECVAM Scientific Advisory Committee, 2016), and has been adopted as OECD TG 442C.

\section{The ARE-Nrf2 luciferase method LuSens}

The ARE-Nrf2 luciferase method covered by KeratinoSens ${ }^{\mathrm{TM}}$ (Natsch et al., 2011) and LuSens (Ramirez et al., 2014, 2016) is described in OECD TG 442D (OECD, 2015a, 2018a). It addresses the second key event of the skin sensitization AOP, namely keratinocyte activation, by assessing the Nrf2-mediated activation of antioxidant response element (ARE)-dependent genes with the help of luciferase. Skin sensitizers have been reported to induce genes that are regulated by the ARE. Small electrophilic substances such as skin sensitizers can act on the sensor protein Keap1 (Kelch-like ECH-associated protein 1) by, e.g., covalent modification of its cysteine residue, resulting in its dissociation from the transcription factor Nrf2 (nuclear factor-erythroid 2-related factor 2). The LuSens test method (Ramirez et al., 2014, 2016) makes use of an immortalized adherent cell line derived from human keratinocytes stably harboring a luciferase reporter gene under the control of the ARE. The luciferase signal reflects the activation of endogenous Nrf2 dependent genes by sensitiz- 
ers (OECD, 2018a). If the statistically significant fold induction (FI) of the luciferase activity is above 1.5 , at relative cell viabilities of at least $70 \%$, the test result is positive and the substance is considered to have keratinocyte activating potential.

\section{The human Cell Line Activation Test (hCLAT)}

The h-CLAT method (Ashikaga et al., 2006, 2010; Sakaguchi et al., 2006, 2010) has recently been validated by ECVAM and is described in OECD TG 442E (OECD, 2018b). It addresses the third key event of the skin sensitization AOP, namely dendritic cell activation. Induction of the expression of the cell surface markers CD54 and CD86 is measured after treatment with a test substance relative to concurrent vehicle controls in immortalized human monocytic leukemia THP-1 cells as a surrogate of dendritic cells. These surface molecules are typical markers of monocytic THP-1 activation and may mimic dendritic cell activation, which plays a critical role in T-cell priming. The changes in surface marker expression are measured by flow cytometry following cell staining with fluorochrome-tagged antibodies. If at least a two-fold induction of the CD54 expression and/or a 1.5-fold induction of CD86 expression are observed at relative cell viabilities of at least $50 \%$, the test result is positive and the substance is considered to indicate a dendritic cell activating potential.

\section{The " 2 out of 3 " ITS}

The "2 out of 3" ITS (Bauch et al., 2012; Urbisch et al., 2015) combines test results from the DPRA, ARE-Nrf2 luciferase method (covered by either LuSens or KeratinoSens ${ }^{\mathrm{TM}}$ ), and the h-CLAT. It is the first case study in the recent report of the OECD describing defined approaches (DA) to be used within integrated approaches to testing and assessment (IATA) for skin sensitization (OECD, 2016). Equal weights are attached to each of the non-animal testing methods, which capture the three key events of the skin sensitization AOP. The overall classification of a substance is determined by the majority of concordant test results from the DPRA, LuSens or KeratinoSens ${ }^{\mathrm{TM}}$, and the h-CLAT.

\subsection{Methodological approach for quantifying the borderline range around a testing method's classification threshold}

For the classification of a test substance's sensitization potential, both animal and non-animal testing methods apply prediction models using defined threshold values that dichotomize continuous experimental results into binary, i.e., positive and negative, outcomes (Hoffmann and Hartung, 2005; van der Schouw et al., 1995; Leontaridou et al., 2017). Comparing experimental results obtained with a non-animal testing method with animal or human data allows quantifying the fractions of substances revealing true positive (TP), true negative (TN), false positive (FP), or false negative (FN) results (Krzanowski and Hand, 2009). Following this, a non-animal testing method's predictive accuracy, i.e., sensitivity, specificity, and concordance, can be determined. Predictive accuracy metrics specify a non-animal testing method's ability to correctly classify an unknown substance compared to the reference animal test, e.g., the LLNA (OECD, 2010).

Following our previous work (Leontaridou et al., 2017), the $\mathrm{BR}$ of a testing method denotes the symmetric range of one pooled standard deviation $\left(S D_{p}\right)$ on both sides of a testing method's classification threshold $T$, pooled across substances and concentrations used:

$B R=\left\{T-S D_{p}, T+S D_{p}\right\}$.

For a given testing method, the $S D_{p}$ of experimental results retrieved from testing different substances and concentrations is defined as:

Tab. 1: Range of test results around the classification threshold used to calculate the pooled standard deviation $S D_{p}$, the borderline range of each testing method and the "2 out of 3 " ITS, the size of the entire sample and of the adapted sample of substances after excluding the number (percentage) of substances identified as borderline

\begin{tabular}{|c|c|c|c|c|c|}
\hline & $\begin{array}{l}\text { Range of test } \\
\text { results around } T \\
\text { for calculating the } \\
\text { pooled standard } \\
\text { deviation } S_{p_{p}}^{*}\end{array}$ & $\mathrm{SD}_{\mathrm{p}}$ and $\mathrm{BR}$ & $\begin{array}{l}\text { Entire sample } \\
\text { (including } \\
\text { borderline } \\
\text { substances) }\end{array}$ & $\begin{array}{l}\text { Adapted sample } \\
\text { (excluding } \\
\text { borderline } \\
\text { substances) }\end{array}$ & $\begin{array}{l}\text { Number and } \\
\text { percentage of } \\
\text { borderline } \\
\text { substances }\end{array}$ \\
\hline DPRA & $(3.38 \% \leq \mathrm{MPD} \leq 9.38 \%)$ & $\begin{array}{l}\mathrm{SD}_{p}=1.52 \% \\
\mathrm{MPD}=\{4.86 \%, 7.9 \%\}\end{array}$ & 199 & 179 & $20(10 \%)$ \\
\hline LuSens & $F I \leq 3$ & $\begin{array}{l}\mathrm{SD}_{p}=0.229 \\
\mathrm{FI}=\{1.27,1.73\}\end{array}$ & 79 & 74 & $5(6 \%)$ \\
\hline h-CLAT & $\begin{array}{l}\text { CD54 } F I \leq 3 \\
\text { CD86 } F I \leq 3\end{array}$ & $\begin{array}{l}\text { CD54 Fl: SDp }=0.190 \\
\text { CD86 Fl: SDp }=0.260 \\
\text { CD54 FI }=\{1.81,2.19\} \\
\text { CD86 FI }=\{1.24,1.76\}\end{array}$ & 40 & 32 & $8(20 \%)$ \\
\hline $\begin{array}{l}\text { "2 out of } \\
\text { 3" ITS }\end{array}$ & & & 40 & 36 & $4(10 \%)$ \\
\hline
\end{tabular}

$\mathrm{T}$, classification threshold; $\mathrm{BR}$, borderline range; $\mathrm{SD}_{\mathrm{p}}$, pooled standard deviation; FI, fold induction; CD54 FI, CD86 FI, fold induction of cell surface marker expression; MPD, mean peptide depletion; * The chosen range is the smallest of a set of ranges tested in Leontaridou et al. (2017). Source: Adapted from Leontaridou et al. (2017). 
Tab. 2: Scenarios for assessing the impact of precision uncertainty, varying sample composition and varying sample size on non-animal methods' predictive accuracy metrics

\begin{tabular}{|l|l|l|l|}
\cline { 2 - 4 } \multicolumn{1}{c|}{} & Experimental sample & Randomized sample & Randomized sub-samples \\
\hline $\begin{array}{l}\text { Entire sample } \\
\text { (including borderline } \\
\text { substances) }\end{array}$ & $\begin{array}{l}\text { Scenario 1: Impact of } \\
\text { precision uncertainty on } \\
\text { predictive accuracy metrics }\end{array}$ & $\begin{array}{l}\text { Scenario 2a: Impact of } \\
\text { uncertainty due to varying } \\
\text { sample composition on } \\
\text { predictive accuracy metrics }\end{array}$ & $\begin{array}{l}\text { Scenario 3a: Joint impact of } \\
\text { varying sample composition } \\
\text { and sample size on predictive } \\
\text { accuracy metrics }\end{array}$ \\
\cline { 3 - 5 } & $\begin{array}{l}\text { Adapted sample } \\
\text { (excluding borderline } \\
\text { substances) }\end{array}$ & $\begin{array}{l}\text { varying sample composition } \\
\text { and precision uncertainty on } \\
\text { predictive accuracy metrics }\end{array}$ & $\begin{array}{l}\text { Scenario 3b: Joint impact of } \\
\text { varying sample composition, } \\
\text { sample size, and precision } \\
\text { uncertainty on predictive } \\
\text { accuracy metrics }\end{array}$ \\
\hline
\end{tabular}

$S D_{p}=\sqrt{\frac{\sum_{i=1}^{n} \sum_{j=1}^{k_{i}}\left(r_{i, j}-1\right) * \sigma_{i, j}^{2}}{\sum_{i=1}^{n} \sum_{j=1}^{k_{i}}\left(r_{i, j}-1\right)}}$,

where $\sigma_{i, j}^{2}$ is the variance of results for substance $i$ and concentration $j . r_{i, j}$ denotes the number of replicates per substance $i$ and concentration $j(j=1-k i)$. The variance of experimental results is given by

$\sigma_{i, j}^{2}=\frac{\sum_{l=1}^{r_{i, j}}\left(y_{i, j, l}-\bar{y}_{i, j}\right)^{2}}{\left(r_{i, j}-1\right)}$,

which acknowledges that for a certain concentration different replicates can be generated. $y_{i, j, l}$ denotes a particular test result of substance $i$, concentration $j$ and replicate $l$, and $\bar{y}_{i, j}$ is the arithmetic mean of test results for substance $i$ and concentration $j$.

Using experimental results for the DPRA, LuSens, the h-CLAT, and for the " 2 out of 3" ITS published in Bauch et al. (2012) and Urbisch et al. (2015), we identified the substances yielding test results within the BR of these methods and the " 2 out of 3" ITS (Leontaridou et al., 2017). Table 1 presents the parameter values for calculating the $\mathrm{BR}$ and the number of substances yielding test results falling into the BR.

\subsection{Scenarios for analyzing the impact of precision uncertainty on the non-animal methods' predictive accuracy}

To examine the impact of limited precision, sample size, and sample composition on classification bias of non-animal testing methods, we defined different scenarios, which are summarized in Table 2.

First, we determined sensitivity (Eq. 4), specificity (Eq. 5), and concordance (Eq. 6) using experimental results from substance samples tested with the DPRA, LuSens, the h-CLAT, and the "2 out of 3" ITS, based on LLNA (Bauch et al., 2012; Natsch et al., 2013; Urbisch et al., 2015).

$$
\begin{aligned}
& \text { Se }[\%]=\frac{T P}{T P+F N} * 100, \\
& S p[\%]=\frac{T N}{T N+F P} * 100,
\end{aligned}
$$

$\operatorname{Con}[\%]=\frac{T P+T N}{T P+T N+F P+F N} * 100$.

The composition of experimental samples, as well as the test results compared to LLNA and human reference data, are documented in Table S1-S41. Accuracy metrics were derived from experimental test results for the full sample (i.e., including the borderline substances) and for the adapted sample (i.e., excluding the borderline substances) following the standard approach discussed in Cooper et al. (1979). The difference in accuracy metrics between the full and the adapted sample illustrates the impact of precision uncertainty (Scenario 1 in Tab. 2).

Second, accuracy metrics were calculated for randomized samples. Randomization was achieved by applying non-parametric standard bootstrap resampling analysis (Tab. 3). This method was used earlier by Worth and Cronin (2001b) to assess the variability of Draize tissue scores. Our study applies a similar approach but focuses on assessing the combined impact of varying sample composition and limited precision on the non-animal methods' accuracy. The bootstrap resampling analysis was conducted in Microsoft Excel. We determined accuracy metrics for the full sample (Scenario $2 \mathrm{a}$ in Tab. 2) and for the adapted sample (Scenario $2 \mathrm{~b}$ in Tab. 2). The difference in accuracy metrics between the two cases illustrates the joint impact of sample composition and precision uncertainty on predictive accuracy metrics.

For every non-animal method and the " 2 out of 3" ITS, a set of $y=10,000$ randomized samples (Efron and Tibshirani, 1993; Ostaszewski and Rempala, 2000) was created by random replacement of the binary classifications obtained from experimental test results (Tab. 3, Step 1). The number of substances in randomized samples, denoted $k$, was equal to the number of substances in the full and adapted experimental sample (Column 4 and 5 in Tab. 1). Randomized samples were assumed to be independent and identically distributed (Wehrens et al., 2000). For all randomized samples we determined sensitivity, specificity, and concordance (Se*, Sp* and Con*, respectively). This revealed non-parametric distributions of sensitivity, specificity, and concordance for the full sample and for the adapted sample (see also Tab. 3, Step 2 and 3). We de-

1 doi:10.14573/altex.1810111s 
termined the mean and the standard deviation $(S D)$ for every distribution according to Eq. (7) and (8):

$$
\begin{aligned}
& \text { Mean }_{a}=\frac{\sum_{1}^{y} a_{y}}{y}, \\
& S D=\sqrt{\frac{\sum_{1}^{y}\left(a_{y}-\text { Mean }_{a}\right)}{y-1}},
\end{aligned}
$$

with a denoting the accuracy metric determined from the randomized sample, and $y$ denoting the number of random samples $(y=10,000)$.
In addition, we calculated confidence limits using the simple percentile method. Specifically, the $95 \%$ confidence interval (95\% CI) was determined by the value corresponding to the $2.5 \%$ and $97.5 \%$ percentile in the bootstrap distribution of sensitivity, specificity, and concordance, respectively (Tab. 3, Step 4).

Third, we assessed accuracy metrics for randomized sub-samples of varying sizes in order to analyze the joint impact of uncertainty in sample size and composition, and of precision uncertainty on the non-animal testing methods' accuracy (Scenario 3a and $3 \mathrm{~b}$ in Tab. 2). Following the procedure for bootstrap resampling

Tab. 3: Steps for conducting non-parametric standard bootstrap resampling analysis

\begin{tabular}{|l|l|}
\hline Step & Description \\
\hline Step 1 & $\begin{array}{l}\text { Bootstrap resampling with random replacement of experimental test results from the individual non-animal testing methods } \\
\text { and the "2 out of 3" ITS. }\end{array}$ \\
\hline Step 2 & Quantification of sensitivity, specificity and concordance for the bootstrap sample (a). \\
\hline Step 3 & $y$-fold repetition of step 1 and 2; $y=10,000$. \\
\hline Step 4 & $\begin{array}{l}\text { Calculation of the mean, the standard deviation, and the 95\% confidence interval of the distributions obtained for } \\
\text { sensitivity, specificity and concordance. }\end{array}$ \\
\hline
\end{tabular}

\begin{tabular}{|c|c|c|}
\hline \multicolumn{3}{|l|}{ DPRA } \\
\hline Sample including borderline substances $(k=199)$ & $\begin{array}{l}\text { Sensitivity [\%] } \\
\text { Specificity [\%] } \\
\text { Concordance [\%] }\end{array}$ & $\begin{array}{l}76 \\
72 \\
75\end{array}$ \\
\hline Sample excluding borderline substances $(k=179)$ & $\begin{array}{l}\text { Sensitivity [\%] } \\
\text { Specificity [\%] } \\
\text { Concordance [\%] }\end{array}$ & $\begin{array}{l}80 \\
74 \\
78\end{array}$ \\
\hline \multicolumn{3}{|l|}{ LuSens } \\
\hline Sample including borderline substances $(k=79)$ & $\begin{array}{l}\text { Sensitivity [\%] } \\
\text { Specificity [\%] } \\
\text { Concordance [\%] }\end{array}$ & $\begin{array}{l}75 \\
70 \\
73\end{array}$ \\
\hline Sample excluding borderline substances $(k=74)$ & $\begin{array}{l}\text { Sensitivity [\%] } \\
\text { Specificity [\%] } \\
\text { Concordance [\%] }\end{array}$ & $\begin{array}{l}77 \\
69 \\
74 \\
\end{array}$ \\
\hline \multicolumn{3}{|l|}{$\mathrm{h}$-CLAT } \\
\hline Sample including borderline substances $(k=40)$ & $\begin{array}{l}\text { Sensitivity [\%] } \\
\text { Specificity [\%] } \\
\text { Concordance [\%] }\end{array}$ & $\begin{array}{l}88 \\
87 \\
88\end{array}$ \\
\hline Sample excluding borderline substances $(k=32)$ & $\begin{array}{l}\text { Sensitivity [\%] } \\
\text { Specificity [\%] } \\
\text { Concordance [\%] }\end{array}$ & $\begin{array}{l}82 \\
87 \\
84\end{array}$ \\
\hline \multicolumn{3}{|l|}{ "2 out of 3" ITS } \\
\hline Sample including borderline substances $(k=40)$ & $\begin{array}{l}\text { Sensitivity [\%] } \\
\text { Specificity [\%] } \\
\text { Concordance [\%] }\end{array}$ & $\begin{array}{l}85 \\
93 \\
88\end{array}$ \\
\hline Sample excluding borderline substances $(k=36)$ & $\begin{array}{l}\text { Sensitivity [\%] } \\
\text { Specificity [\%] } \\
\text { Concordance [\%] }\end{array}$ & $\begin{array}{l}82 \\
93 \\
86\end{array}$ \\
\hline
\end{tabular}

Tab. 4: Impact of precision uncertainty on predictive accuracy metrics of non-animal testing methods and the " 2 out of 3 " ITS*

*Experimental data compared to LLNA reference data. Source: Own calculations. 
outlined in Table 3, we calculated the mean, the SD and the 95\% CI of the predictive accuracy metrics for each sub-sample, including and excluding borderline substances. Sub-samples consisted of $k=10 ; 50 ; 100$; and 150 substances for the DPRA (with random replacement from the experimental sample consisting of 199 substances), $k=10 ; 20 ; 40$ and 60 substances for LuSens (with random replacement from the experimental sample consisting of 79 substances), and of $k=10$ and 20 substances for the h-CLAT and the " 2 out of 3" ITS (with random replacement from the experimental samples consisting of 40 substances), respectively.

\section{Results}

This section presents the results from analyzing the impact of borderline substances on accuracy metrics of DPRA, LuSens, h-CLAT, and the " 2 out of 3" ITS, using the LLNA as reference test. Tables S5-S7 ${ }^{1}$ show results obtained from experimental samples compared to human data.

\subsection{Impact of precision uncertainty}

Excluding test results within the BR increased sensitivity, specificity, and the concordance of the DPRA (with 20 out of 199 substances yielding results in the BR), but had little effect on the predictive accuracy metrics of the LuSens (with 5 out of 79 substances with results in the BR) (Tab. 4). Sensitivity and the concordance of the h-CLAT decreased (with 8 out of 40 substances with results in the BR) after excluding borderline substances. Likewise, excluding results falling in the BR for the " 2 out of 3 " ITS decreased sensitivity and concordance (86\% to $82 \%$ with 4 out of 40 substances in the BR). Note that the " 2 out of 3 " ITS reveals fewer borderline substances than the h-CLAT because the final classification is based on results from three methods. For example, LuSens and DPRA results that are not in the BR may be sufficient to conclude a " 2 out of 3 " ITS result to be outside the $\mathrm{BR}$ even if the result obtained in the h-CLAT, as a third assay, is within the BR.

\subsection{Joint impact of precision uncertainty and varying sample composition}

We received a distribution of accuracy metrics for each non-animal testing method as a result of the non-parametric bootstrapping procedure. We found predictive accuracy metrics to be normally distributed for the DPRA and LuSens, while we observed a left-skewed distribution of accuracy metrics for the h-CLAT and the "2 out of 3" ITS approach.

Randomization causes the sample composition to differ. Consequently, the number of borderline substances differs as well.
The minimum and maximum number of substances in the adapted samples is shown in Table 5.

Table 6 presents the mean and the SD (Column 3) and the 95\% CI (Column 4) revealed from the distributions of sensitivity, specificity, and concordance values. The table shows the mean, the SD and the $95 \%$ confidence interval of accuracy metrics obtained from randomized samples including borderline samples (see Scenario 2a in Tab. 2), documenting the impact of variations in sample composition on a method's predictivity. Furthermore, results from adapted samples (see Scenario $2 b$ in Tab. 2) are presented. This illustrates the joint impact of a varying sample composition, and precision uncertainty. While the general pattern of impacts on mean accuracy metrics does not change, the confidence intervals of the accuracy metrics shift in case of the DPRA and LuSens, and increase or decrease in case of the h-CLAT and the " 2 out of 3 " ITS.

\subsection{Joint impact of varying sample composition and sample size}

We created sub-samples of different sizes in order to analyze the joint impact of varying sample size and sample composition on predictive accuracy metrics. Table 7 presents the mean, the standard deviation, and the confidence interval of all accuracy metrics revealed from randomized samples of different sizes.

\subsection{Joint impact of varying sample composition and sample size in combination with precision uncertainty} Finally, the parameters revealed for the distributions of sensitivity, specificity, and concordance from adapted samples (see Scenario $3 \mathrm{~b}$ in Tab. 2) offer insight into the joint impact of varying sample size variation, sample composition, and precision uncertainty on predictive accuracy metrics (Tab. 8). Note that the number of borderline substances within sub-samples could differ due to the randomization of experimental samples. Thus, similar to Table 5, we can determine the minimum and maximum number of substances for all subsamples after excluding borderline substances (Column 1 of Table 8). Distribution parameters of accuracy metrics (Columns 2-4) capture the range of sample sizes per sub-sample.

\section{Discussion}

\subsection{Impact of uncertainty due to limited precision}

Acknowledging that borderline substances cannot be classified as "positive" or "negative", we expected that predictive accuracy metrics derived from the full samples (i.e., including borderline substances) would differ from those of adapted samples (i.e.,

Tab. 5: Minimum and maximum number of substances $(k)$ in randomized samples resulting from bootstrap resampling after borderline substances were excluded

\begin{tabular}{|l|l|l|l|l|}
\hline Randomized sample size $(\boldsymbol{k})$ & DPRA & LuSens & h-CLAT & "2 out of 3" ITS \\
\hline Min & 160 & 65 & 33 & 27 \\
\hline Max & 194 & 79 & 40 & 40 \\
\hline
\end{tabular}

Source: Own calculations. 
Tab. 6: Mean, standard deviation (SD) and 95\% confidence intervals of accuracy metrics of the DPRA, LuSens, the h-CLAT, and the " 2 out of 3 " ITS determined from randomised samples*

\begin{tabular}{|c|c|c|c|}
\hline & & \\
\hline & & Mean \pm SD & $95 \% \mathrm{Cl}$ \\
\hline \multicolumn{4}{|l|}{ DPRA } \\
\hline Sample including borderline substances & $\begin{array}{l}\text { Sensitivity [\%] } \\
\text { Specificity [\%] } \\
\text { Concordance [\%] }\end{array}$ & $\begin{array}{l}76 \pm 4 \\
72 \pm 6 \\
75 \pm 3\end{array}$ & $\begin{array}{l}(69 ; 83) \\
(60 ; 83) \\
(69 ; 81)\end{array}$ \\
\hline Sample excluding borderline substances & $\begin{array}{l}\text { Sensitivity [\%] } \\
\text { Specificity [\%] } \\
\text { Concordance [\%] }\end{array}$ & $\begin{array}{l}80 \pm 4 \\
74 \pm 6 \\
78 \pm 3\end{array}$ & $\begin{array}{l}(73 ; 86) \\
(62 ; 86) \\
(72 ; 84)\end{array}$ \\
\hline \multicolumn{4}{|l|}{ LuSens } \\
\hline Sample including borderline substances & $\begin{array}{l}\text { Sensitivity [\%] } \\
\text { Specificity [\%] } \\
\text { Concordance [\%] }\end{array}$ & $\begin{array}{l}75 \pm 6 \\
71 \pm 9 \\
73 \pm 5\end{array}$ & $\begin{array}{l}(63 ; 87) \\
(53 ; 88) \\
(64 ; 83)\end{array}$ \\
\hline Sample excluding borderline substances & $\begin{array}{l}\text { Sensitivity [\%] } \\
\text { Specificity [\%] } \\
\text { Concordance [\%] }\end{array}$ & $\begin{array}{l}77 \pm 6 \\
69 \pm 9 \\
74 \pm 5\end{array}$ & $\begin{array}{l}(65 ; 89) \\
(51 ; 87) \\
(64 ; 84)\end{array}$ \\
\hline \multicolumn{4}{|l|}{ h-CLAT } \\
\hline Sample including borderline substances & $\begin{array}{l}\text { Sensitivity [\%] } \\
\text { Specificity [\%] } \\
\text { Concordance [\%] }\end{array}$ & $\begin{array}{l}88 \pm 7 \\
87 \pm 9 \\
87 \pm 5\end{array}$ & $\begin{array}{l}(74 ; 100) \\
(67 ; 100) \\
(78 ; 98)\end{array}$ \\
\hline Sample excluding borderline substances & $\begin{array}{l}\text { Sensitivity [\%] } \\
\text { Specificity [\%] } \\
\text { Concordance [\%] }\end{array}$ & $\begin{array}{l}82 \pm 9 \\
87 \pm 9 \\
84 \pm 6\end{array}$ & $\begin{array}{l}(62 ; 100) \\
(67 ; 100) \\
(71 ; 96)\end{array}$ \\
\hline \multicolumn{4}{|l|}{ “2 out of 3" ITS } \\
\hline Sample including borderline substances & $\begin{array}{l}\text { Sensitivity [\%] } \\
\text { Specificity [\%] } \\
\text { Concordance [\%] }\end{array}$ & $\begin{array}{l}85 \pm 7 \\
93 \pm 7 \\
87 \pm 5\end{array}$ & $\begin{array}{l}(70 ; 96) \\
(77 ; 100) \\
(78 ; 98)\end{array}$ \\
\hline Sample excluding borderline substances & $\begin{array}{l}\text { Sensitivity [\%] } \\
\text { Specificity [\%] } \\
\text { Concordance [\%] }\end{array}$ & $\begin{array}{l}82 \pm 8 \\
93 \pm 7 \\
86 \pm 6\end{array}$ & $\begin{array}{l}(64 ; 96) \\
(77 ; 100) \\
(74 ; 97)\end{array}$ \\
\hline
\end{tabular}

${ }^{*}$ Experimental data compared to LLNA reference data. Source: Own calculations.

excluding borderline substances). Indeed, our results show that accounting for precision uncertainty changed accuracy metrics of the DPRA, LuSens, the h-CLAT, and the " 2 out of 3 " ITS approach (Tab. 4). However, this impact was not symmetric across individual non-animal testing methods and the " 2 out of 3 " ITS. In particular, whereas sensitivity, specificity, and concordance increased after the exclusion of borderline substances from experimental samples for the DPRA, sensitivity and concordance derived from test results compared to the LLNA decreased considerably for the h-CLAT and remained almost unchanged when derived from test results compared to human data (see Tab. S711). We observed a small increase of sensitivity for LuSens, but no change of specificity and even a slight decrease of the concordance. For the " 2 out of 3 " ITS approach, we found an increase of sensitivity, specificity, and concordance when assessed with borderline substances compared to the LLNA. Similar results were observed for the accuracy metrics when test results of the non-animal testing methods were compared to human data (see Tab. $\left.S 7^{1}\right)$.
For individual non-animal testing methods, the size and direction of the impact on accuracy metrics also depends on the composition of experimental samples and whether test results of borderline substances are above or below the classification threshold. If, as in the case of the DPRA, more borderline substances revealed results below the classification threshold (thus they would be classified as "negative" when ignoring the BR), excluding these substances increased the fraction of substances classified as "positive", which in turn caused an increase of sensitivity. In contrast, test results of substances providing results in the BR of the h-CLAT were all above the classification threshold (Leontaridou et al., 2017). Excluding these substances in order to correct for ambiguous classifications decreased sensitivity for the experimental sample with the LLNA as reference test (see Tab. 4 and 6). In addition, since accounting for the BR changed the fractions of TP and FN classifications of the substances remaining in the sample, we observed a slight decrease of concordance. In case of LuSens, only few substances were identified as borderline in the experimental sample (4 out 
Tab. 7: Mean, SD and $95 \% \mathrm{Cl}$ of accuracy metrics revealed for the DPRA, LuSens, the h-CLAT, and the "2 out of 3 " ITS for randomized full samples of different sizes (i.e., including borderline substances)*

\begin{tabular}{|c|c|c|c|c|c|c|}
\hline \multirow[b]{2}{*}{ Sample size $(k)$} & \multicolumn{2}{|c|}{ Sensitivity [\%] } & \multicolumn{2}{|c|}{ Specificity [\%] } & \multicolumn{2}{|c|}{ Concordance [\%] } \\
\hline & Mean \pm SD & $95 \% \mathrm{Cl}$ & Mean \pm SD & $95 \% \mathrm{Cl}$ & Mean \pm SD & $95 \% \mathrm{Cl}$ \\
\hline \multicolumn{7}{|l|}{ DPRA } \\
\hline 10 & $76 \pm 16$ & $(43 ; 100)$ & $71 \pm 29$ & $(0 ; 100)$ & $75 \pm 14$ & $(50 ; 100)$ \\
\hline 50 & $76 \pm 7$ & $(61 ; 90)$ & $72 \pm 12$ & $(46 ; 93)$ & $75 \pm 6$ & $(62 ; 86)$ \\
\hline 100 & $76 \pm 5$ & $(66 ; 86)$ & $72 \pm 8$ & $(55 ; 87)$ & $75 \pm 4$ & $(66 ; 83)$ \\
\hline 150 & $76 \pm 4$ & $(68 ; 84)$ & $72 \pm 7$ & $(57 ; 84)$ & $75 \pm 4$ & $(68 ; 81)$ \\
\hline $199^{a}$ & $76 \pm 4$ & $(69 ; 83)$ & $72 \pm 6$ & $(60 ; 83)$ & $75 \pm 3$ & $(69 ; 81)$ \\
\hline \multicolumn{7}{|l|}{ LuSens } \\
\hline 10 & $75 \pm 17$ & $(38 ; 100)$ & $70 \pm 28$ & $(0 ; 100)$ & $73 \pm 14$ & $(40 ; 100)$ \\
\hline 20 & $75 \pm 12$ & $(50 ; 94)$ & $70 \pm 19$ & $(33 ; 100)$ & $73 \pm 10$ & $(55 ; 90)$ \\
\hline 40 & $75 \pm 9$ & $(57 ; 91)$ & $70 \pm 13$ & $(44 ; 92)$ & $73 \pm 7$ & $(60 ; 88)$ \\
\hline 60 & $75 \pm 7$ & $(61 ; 88)$ & $70 \pm 10$ & $(50 ; 89)$ & $73 \pm 6$ & $(62 ; 85)$ \\
\hline $79^{a}$ & $77 \pm 6$ & $(65 ; 89)$ & $69 \pm 9$ & $(51 ; 87)$ & $74 \pm 5$ & $(64 ; 84)$ \\
\hline \multicolumn{7}{|l|}{ h-CLAT } \\
\hline 10 & $88 \pm 14$ & $(56 ; 100)$ & $87 \pm 19$ & $(40 ; 100)$ & $87 \pm 10$ & $(60 ; 100)$ \\
\hline 20 & $88 \pm 9$ & $(67 ; 100)$ & $87 \pm 13$ & $(57 ; 100)$ & $87 \pm 7$ & $(70 ; 100)$ \\
\hline $40^{a}$ & $88 \pm 7$ & $(74 ; 100)$ & $87 \pm 9$ & $(67 ; 100)$ & $75 \pm 5$ & $(64 ; 84)$ \\
\hline \multicolumn{7}{|l|}{ "2 out of 3" ITS } \\
\hline 10 & $84 \pm 15$ & $(50 ; 100)$ & $93 \pm 15$ & $(50 ; 100)$ & $87 \pm 10$ & $(60 ; 100)$ \\
\hline 20 & $85 \pm 10$ & $(63 ; 100)$ & $93 \pm 10$ & $(67 ; 100)$ & $88 \pm 7$ & $(70 ; 100)$ \\
\hline $40^{a}$ & $85 \pm 7$ & $(70 ; 96)$ & $93 \pm 7$ & $(77 ; 100)$ & $87 \pm 5$ & $(78 ; 98)$ \\
\hline
\end{tabular}

* Experimental data compared to LLNA reference data. ${ }^{\text {a }}$ Size of the full experimental sample. Source: Own calculations.

of 79 substances, i.e., 5\%; Leontaridou et al., 2017). Due to the stringent acceptance criteria and the prediction model of LuSens (Ramirez et al., 2014, 2016), excluding these substances had only a marginal impact on predictive accuracy metrics. Finally, we found a slight decrease of sensitivity and concordance, and no change of specificity of the " 2 out of 3 " ITS. This can be explained by considering that the prediction model of the " 2 out of 3" ITS bases the overall conclusion on the skin sensitization potential of a substance on at least two concordant test results from the DPRA, LuSens or the h-CLAT, and assigns equal weights to each testing method. This balances the impact of precision uncertainty on predictive accuracy metrics observed for the individual non-animal testing methods.

\subsection{Impact of precision uncertainty and varying sample composition}

Determining accuracy metrics from randomized samples allows specifying their variation depending on the sample composition (Scenario 2a in Tab. 2). The mean of the distributions of accuracy metrics corresponded to the values observed for the determin- istic experimental samples for all individual non-animal testing methods and the "2 out of 3" ITS (Tab. 6).

Our results illustrate that accounting for precision uncertainty in combination with randomized sampling did not affect the mean of accuracy metrics. In case of the h-CLAT and the " 2 out of 3" ITS, we observe a slight increase of the SD of accuracy metrics - and thus a small increase of the overall uncertainty of these metrics. Furthermore, we found the $95 \%$ confidence interval to slightly shift for most accuracy metrics.

\subsection{Impact of uncertainty due to sample size}

Our results illustrate that increasing sample size (Scenario $2 \mathrm{~b}$ in Tab. 2) decreased the variability of predictive accuracy metrics expressed by the SD (Tab. 7). This was found for all individual non-animal methods and the " 2 out of 3 " ITS. More specifically, for individual methods and the " 2 out of 3 " ITS, the SD of accuracy metrics from randomized sub-samples was up to four times higher than the SD obtained from randomized full samples. Note that both randomized full and sub-samples still include borderline substances. Furthermore, the $95 \%$ CI of predictive accura- 
Tab. 8: Mean, SD, and 95\% Cl of accuracy metrics revealed for the DPRA, LuSens, the h-CLAT, and the "2 out of 3" ITS for randomized adapted samples (i.e., excluding borderline substances)*

\begin{tabular}{|c|c|c|c|c|c|c|}
\hline \multirow[b]{2}{*}{$\begin{array}{l}\text { Max. and min. number of substances in } \\
\text { adapted, randomized sub-samples }\end{array}$} & \multicolumn{2}{|c|}{ Sensitivity [\%] } & \multicolumn{2}{|c|}{ Specificity [\%] } & \multicolumn{2}{|c|}{ Concordance [\%] } \\
\hline & Mean \pm SD & $95 \% \mathrm{Cl}$ & Mean \pm SD & $95 \% \mathrm{Cl}$ & Mean \pm SD & $95 \% \mathrm{Cl}$ \\
\hline \multicolumn{7}{|l|}{ DPRA } \\
\hline $6-10(k=10)^{\mathrm{a}}$ & $80 \pm 16$ & $(43 ; 100)$ & $74 \pm 30$ & $(0 ; 100)$ & $78 \pm 14$ & $(50 ; 100)$ \\
\hline $37-50(k=50)^{\mathrm{a}}$ & $80 \pm 7$ & $(65 ; 93)$ & $74 \pm 13$ & $(47 ; 95)$ & $78 \pm 6$ & $(66 ; 89)$ \\
\hline $89-99(k=100)^{\mathrm{a}}$ & $80 \pm 5$ & $(70 ; 89)$ & $74 \pm 9$ & $(56 ; 90)$ & $78 \pm 4$ & $(69 ; 87)$ \\
\hline $121-146(k=150)^{\mathrm{a}}$ & $80 \pm 4$ & $(72 ; 88)$ & $74 \pm 7$ & $(60 ; 88)$ & $78 \pm 4$ & $(71 ; 85)$ \\
\hline $160-194(k=199)^{a}$ & $80 \pm 4$ & $(73 ; 86)$ & $74 \pm 6$ & $(62 ; 86)$ & $78 \pm 3$ & $(72 ; 84)$ \\
\hline \multicolumn{7}{|l|}{ LuSens } \\
\hline $4-10(k=10)^{a}$ & $77 \pm 18$ & $(40 ; 100)$ & $69 \pm 29$ & $(0 ; 100)$ & $75 \pm 14$ & $(44 ; 100)$ \\
\hline $14-20(k=20)^{\mathrm{a}}$ & $77 \pm 12$ & $(50 ; 100)$ & $69 \pm 19$ & $(30 ; 100)$ & $74 \pm 10$ & $(53 ; 94)$ \\
\hline $30-40(k=40)^{\mathrm{a}}$ & $77 \pm 8$ & $(59 ; 92)$ & $69 \pm 13$ & $(42 ; 93)$ & $74 \pm 7$ & $(60 ; 87)$ \\
\hline $48-60(k=60)^{\mathrm{a}}$ & $77 \pm 7$ & $(63 ; 90)$ & $69 \pm 11$ & $(47 ; 89)$ & $74 \pm 6$ & $(63 ; 85)$ \\
\hline $65-79(k=79)^{\mathrm{a}}$ & $78 \pm 6$ & $(65 ; 89)$ & $69 \pm 9$ & $(50 ; 86)$ & $75 \pm 5$ & $(64 ; 84)$ \\
\hline \multicolumn{7}{|l|}{ h-CLAT } \\
\hline $6-10(k=10)^{\mathrm{a}}$ & $82 \pm 20$ & $(33 ; 100)$ & $87 \pm 20$ & $(33 ; 100)$ & $84 \pm 13$ & $(56 ; 100)$ \\
\hline $7-20(k=20)^{\mathrm{a}}$ & $82 \pm 13$ & $(50 ; 100)$ & $87 \pm 13$ & $(57 ; 100)$ & $84 \pm 9$ & $(65 ; 100)$ \\
\hline $33-40(k=40)^{\mathrm{a}}$ & $82 \pm 9$ & $(62 ; 100)$ & $87 \pm 9$ & $(67 ; 100)$ & $84 \pm 6$ & $(71 ; 96)$ \\
\hline \multicolumn{7}{|l|}{ "2 out of 3" ITS } \\
\hline $6-10(k=10)^{a}$ & $81 \pm 18$ & $(40 ; 100)$ & $93 \pm 15$ & $(50 ; 100)$ & $86 \pm 12$ & $(60 ; 100)$ \\
\hline $12-20(k=20)^{\mathrm{a}}$ & $82 \pm 12$ & $(56 ; 100)$ & $93 \pm 10$ & $(67 ; 100)$ & $86 \pm 8$ & $(68 ; 100)$ \\
\hline $27-40(k=40)^{a}$ & $82 \pm 8$ & $(64 ; 96)$ & $93 \pm 7$ & $(77 ; 100)$ & $86 \pm 6$ & $(74 ; 97)$ \\
\hline
\end{tabular}

* Experimental data compared to LLNA reference data. ${ }^{a} k$ denotes the sample size of sub-samples including borderline substances.

Source: Own calculations.

cy metrics was considerably larger, implying that for very small sample sizes a robust prediction of predictive accuracy metrics cannot be provided. Similar results were found for the adapted samples (i.e., excluding borderline substances, see Tab. 8)

Finally, for the DPRA we observed that the SD and the $95 \%$ CI values decreased for sample sizes up to $k=100$ substances, but remained fairly stable at samples sizes of $k \geq 100$ substances, irrespective of whether borderline substances were included or excluded. Our findings suggest, therefore, that uncertainty due to varying sample size (and composition) is large and highly dependent on the actual sample size (and composition) when derived from samples containing $k \leq 50$ substances (for the DPRA; the uncertainty was somewhat smaller with the LuSens and h-CLAT). This may have implications for the interpretation of predictive accuracy metrics presented in other studies. For instance, experimental samples used to validate the DPRA and the h-CLAT included 21 and 24 substances, respectively. In both validation reports substances were tested three times with the DPRA (in three different labs) (ECVAM, 2012), and four times (in four different labs) with the h-CLAT (ECVAM, 2013). Although the assessment of a non-animal testing method's predictive accuracy is only one component in a validation study, it is an important piece of information for concluding on a testing method's ability to provide correct classifications in relation to a reference test. Our findings may, therefore, stimulate a scientific and a policy debate about (the criteria for defining) minimum sample sizes.

\subsection{Joint impact of uncertainty due to sample size, sample composition, and precision uncertainty}

Uncertainty of sample size in combination with varying sample composition (Scenario 3a in Tab. 2) had only a marginal impact on the mean values of accuracy metrics (Tab. 8). Likewise, by additionally considering precision uncertainty (Scenario $3 \mathrm{~b}$ in Tab. 2), mean accuracy metrics derived from randomized samples differed only marginally from those obtained from the experimental samples used to calculate the predictive accuracy of testing methods (compare Tab. 4 with Tab. 7 and 8). This was ob- 
served in both cases of estimating the predictive accuracy based on the full and the adapted sample. Notwithstanding, confidence limits of accuracy metrics obtained for randomized adapted samples are larger for all individual non-animal methods and the "2 out of 3" ITS. This implies that considering precision uncertainty in combination with uncertainty due to sample size and sample composition increased the overall variability of the non-animal methods' predictive accuracy, while not affecting the means of the distributions of accuracy metrics.

\section{Conclusions}

Non-animal methods are frequently assessed based on their ability to produce results which are concordant with the "gold standard" (often the results of animal studies, ideally adequate human data). This concordance is used as a metric of the method's predictive accuracy (usually given as sensitivity, specificity, and concordance). Yet, the predictive accuracy is not a precise number, but is indistinct owing to different types of uncertainty. This holds for all testing methods, i.e., non-animal methods (often the method under scrutiny) and animal tests (often used as the "gold standard"). Uncertainty in determining the predictive accuracy arises from the precision uncertainty of the testing method, and from uncertainty due to varying composition and varying size of the samples of test substances used to determine these metrics. We analyzed the impact for each type of uncertainty individually and as combined impact using selected non-animal methods for skin sensitization testing as an example. The analysis was applied to samples of substances tested with the DPRA, LuSens, and the h-CLAT, and their combination in an integrated testing strategy, the " 2 out of 3 " ITS.

We find that the impact of uncertainties on the predictive accuracy metrics depends, in particular, on the number of borderline substances within the samples (i.e., the number of substances which would be classified as true positive, true negative, false positive or false negative if the BR is ignored). This affects the mean values of accuracy metrics, but also the variation of the data (indicated by the SD).

It is common practice to characterize and assess a test method's predictive accuracy by calculating the sensitivity, specificity, and concordance. So far, however, uncertainties of these metrics have been largely ignored. This may cause bias in conclusions on an individual testing method's predictive accuracy, and may hamper a meaningful comparative evaluation of predictive accuracy across testing methods. To allow for a more transparent and coherent assessment of different testing methods' predictive performance, we therefore propose to amend accuracy metrics by the following information:

1. Documentation of the borderline range (BR) of the test meth$o d$. To unravel precision uncertainty of testing methods, the BR of a test method needs to be determined and substances with experimental results falling within this range should be identified. A prediction model using thresholds should therefore provide three possible outcomes: positive, negative, and inconclusive (in case of binary classifications).
2. Provide information about the potential over- and under-estimation errors of accuracy metrics. Reporting the SD and 95\% CI of the accuracy metrics is useful to document their overall uncertainty and to provide the area that encompasses the predictive accuracy metrics of testing methods if re-assessed using different experimental samples. The CI of the metrics of both methods may, however, differ and indicate that there is significant difference regarding the robustness of these metrics. Likewise, two test methods may appear to be of different predictive accuracy judged by the mean of the metrics. There may, however, be no significant difference if the CI of the metrics of both methods overlap.

3. Definition of a minimum size and requirements for a balanced composition of the set of data used to estimate the predictive accuracy metrics. For a more coherent assessment of the predictive accuracy of testing methods, experimental samples should be of a sufficient size and composition to adequately represent TP, TN, FP, and FN test results. Ideally, the number of substances that is considered sufficient to determine predictive accuracy metrics should be pre-defined.

We believe that these amendments will provide pertinent information for the regulatory acceptance or rejection of a new testing method as well as the comparison of the predictive performances of two testing methods.

Finally, our analysis does not claim to be exhaustive but should be regarded as a first step into systematically exploring precision uncertainty. This is a prerequisite for more transparent and informative evaluation of testing methods. Based on our findings, further research can go in different directions. For instance, quantifying the borderline range for a broader set of non-animal methods allows for comparative evaluations of precision uncertainty across endpoints. Furthermore, assessments of precision uncertainty must also include the reference data (human or animal data used as "gold standard"). Though being beyond the scope of this paper, exploring the impact of limited precision of the animal test on precision uncertainty of non-animal methods is a next step to allow for comparisons of biases in predictive accuracy between animal and non-animal testing methods.

\section{References}

Agnese, G., Risso, D. and De Flora, S. (1984). Statistical evaluation of inter- and intra-laboratory variations of the Ames test, as related to the genetic stability of Salmonella tester strains. Mutat Res 130, 27-44. doi:10.1016/0165-1161(84)90004-9

Ashikaga, T., Yoshida, Y., Hirota, M. et al. (2006). Development of an in vitro skin sensitization test using human cell lines: The human cell line activation test (h-CLAT): I. Optimization of the h-CLAT protocol. Toxicol In Vitro 20, 767-773. doi:10.1016/j.tiv.2005.10.012

Ashikaga, T., Sakaguchi, H., Sono, S. et al. (2010). A comparative evaluation of in vitro skin sensitisation tests: The human cell-line activation test (h-CLAT) versus the Local Lymph Node Assay (LLNA). Altern Lab Anim 38, 275-284. doi:10.1177/026119291003800403 
Bauch, C., Kolle, S. N., Ramirez, T. et al. (2012). Putting the parts together: Combining in vitro methods to test for skin sensitizing potentials. Regul Toxicol Pharmacol 63, 489-504. doi:10.1016/j.yrtph.2012.05.013

Bruner, L. H., Carr, G. J., Chamberlain, M. and Curren, R. D. (1996). Validation of alternative methods for toxicity testing. Toxicol In Vitro 10, 479-501. doi:10.1016/08872333(96)00028-8

Cooper, J. A., $2^{\text {nd }}$, Saracci, R. and Cole, P. (1979). Describing the validity of carcinogen screening tests. Br J Cancer 39, 87-89. doi:10.1038/bjc.1979.10

Dimitrov, S., Detroyer, A., Piroird, C. et al. (2016). Accounting for data variability, a key factor in in vivo/in vitro relationships: Application to the skin sensitization potency (in vivo LLNA versus in vitro DPRA) example. J Appl Toxicol 36, 1568-1578. doi:10.1002/jat.3318

Dumont, C., Barroso, J., Matys, I. et al. (2016). Analysis of the local lymph node assay (LLNA) variability for assessing the prediction of skin sensitisation potential and potency of chemicals with non-animal approaches. Toxicol In Vitro 34, 220 228. doi:10.1016/j.tiv.2016.04.008

EC - European Commission (2006). Regulation (EC) No 1907/2006 of the European Parliament and the Council of of 18 December 2006 concerning the Registration, Evaluation, Authorisation and Restriction of Chemicals (REACH), establishing a European Chemicals Agency, amending Directive 1999/45/EC and repealing Council Regulation (EEC) No 793/93 and Commission Regulation (EC) No 1488/94 as well as Council Directive 76/769/EEC and Commission Directives 91/155/EEC, 93/67/EEC, 93/105/EC and 2000/21/EC. http:// data.europa.eu/eli/reg/2006/1907/2014-04-10

EC (2009). Regulation (EC) No 1223/2009 of the European Parliament and of the Council of 30 November 2009 on cosmetic products. https://ec.europa.eu/health/sites/health/files/ endocrine_disruptors/docs/cosmetic_1223_2009_regulation en.pdf (accessed April 2019).

ECHA (2016). Guidance on Information Requirements and Chemical Safety Assessment. Chapter R.7a: Endpoint specific guidance. Draft Version 5.0. https://bit.ly/2JvRYhG

ECVAM (2012). Direct Peptide Reactivity Assay (DPRA) ECVAM Validation Study Report.

ECVAM (2013). Human Cell Line Activation Test (h-CLAT) Validation Study Report.

EURL ECVAM Scientific Advisory Committee (2016). ESAC Opinion on the BASF-coordinated Performance Standards-based validation of the LuSens test method for skin sensitisation testing. ESAC Opinion No. 2016-04 of 24 June 2016; EUR 28176 EN. doi:10.2787/170247

Efron, B. and Tibshirani, R. J. (1993). An Introduction to the Bootstrap. London, UK: Chapman and Hall/CRC Monographs on Statistics and Applied Probability.

Gerberick, G. F., Vassallo, J. D., Bailey, R. E. et al. (2004). Development of a peptide reactivity assay for screening contact allergens. Toxicol Sci 81, 332-334. doi:10.1093/toxsci/kfh213

Gerberick, G. F., Vassallo, J. D., Foertsch, L. M. (2007). Quantification of chemical peptide reactivity for screening contact allergens: A classification tree model approach. Toxicol Sci 97, 417-427. doi:10.1093/toxsci/kfm064

Hoffmann, S. and Hartung, T. (2005). Diagnosis: Toxic! - Trying to apply approaches of clinical diagnostics and prevalence in toxicology considerations. Toxicol Sci 85, 422-428. doi:10.1093/toxsci/kfi099

Hoffmann, S. (2015). LLNA variability: An essential ingredient for a comprehensive assessment of non-animal skin sensitization test methods and strategies. ALTEX 32, 379-383. doi:10.14573/altex.1505051

Hothorn, L. A. (2002). Selected biostatistical aspects of the validation of in vitro toxicological assays. Altern Lab Anim 30, Suppl 2, 93-98. doi:10.1177/026119290203002S14

Hothorn, L. A. (2003). Statistics of interlaboratory in vitro toxicological studies. Altern Lab Anim 31, Suppl 1, 43-63. doi:10.1177/026119290303101s03

Kleinstreuer, N. C., Hoffmann, S., Alépée, N. et al. (2018). Non-animal methods to predict skin sensitization (II): An assessment of defined approaches. Crit Rev Toxicol 48, 359-374. doi:10.1080/10408444.2018.1429386

Kolle, S. N., Basketter, D. A., Casati, S. et al. (2013). Performance standards and alternative assays: Practical insights from skin sensitization. Regul Toxicol Pharmacol 65, 278285. doi:10.1016/j.yrtph.2012.12.006

Kolle, S. N., Hill, E., Raabe, H. et al. (2019). Regarding the references for reference chenicals of alternative methods. Toxicol In Vitro 57, 48-53. doi:10.1016/j.tiv.2019.02.007

Krzanowski, W. L. and Hand, D. J. (2009). ROC Curves for Continuous Data. London, UK: CRC Press Taylor \& Francis Group.

Leontaridou, M., Urbisch, D., Kolle, S. et al. (2017). The borderline range of toxicological methods: Quantification and implications for evaluating precision. ALTEX 34, 525-538. doi:10.14573/altex.1606271

Margolin, B. H., Risko, K. J., Shelby, M. D. and Zeiger, E. (1984). Sources of variability in Ames Salmonella typhimurium tester strains: Analysis of the international collaborative study on 'genetic drift'. Mutat Res 130, 11-25. doi:10.1016/0165-1161(84)90003-7

Mehling, A., Eriksson, T., Eltze, T. et al. (2012). Non-animal test methods for predicting skin sensitization potentials. Arch Toxicol 86, 1273-1295. doi:10.1007/s00204-012-0867-6

Natsch, A., Bauch, C., Foertsch, L. et al. (2011). The intra- and inter-laboratory reproducibility and predictivity of the KeratinoSens assay to predict skin sensitizers in vitro: Results of a ring-study in five laboratories. Toxicol In Vitro 25, 733-744. doi:10.1016/j.tiv.2010.12.014

Natsch, A., Ryan, C. A., Foertsch, L. et al. (2013). A dataset on 145 chemicals tested in alternative assays for skin sensitization undergoing prevalidation. J Appl Toxicol 33, 1337-1352. doi:10.1002/jat.2868

OECD (2010). Test No. 429: Skin Sensitisation: Local Lymph Node Assay. OECD Guidelines for the Testing of Chemicals, Section 4. OECD Publishing, Paris. doi:10.1787/ 9789264071100-en

OECD (2015a). Test No. 442C: In Chemico Skin Sensitisation. Direct Peptide Reactivity Assay (DPRA). OECD Guidelines 
for the Testing of Chemicals, Section 4. OECD Publishing, Paris. doi:10.1787/9789264229709-en

OECD (2015b). Performance Standards for Assessment of Proposed Similar or Modified In Vitro Skin Sensitisation ARENrf2 Luciferase Test Methods. Series on Testing and Assessment No. 213. OECD, Paris. http://www.oecd.org/env/ ehs/testing/Performance-Standards-for-Assessment-of-Proposed-Similar-or-Modified-in-vitro-Skin--Sensitisation-ARE-NRF2-Luciferase-Test-Methods.pdf

OECD (2016). Guidance Document on the Reporting of Defined Approaches to be Used Within Integrated Approaches to Testing and Assessment. Task Force on Hazard Assessment. OECD Publishing, Paris. https://read.oecd-ilibrary.org/ environment/guidance-document-on-the-reporting-ofdefined-approaches-to-be-used-within-integrated-approaches -to-testing-and-assessment_9789264274822-en\#page1

OECD (2018a). Test No. 442D: In Vitro Skin Sensitisation: ARE-Nrf2 Luciferase Test Method. OECD Guidelines for the Testing of Chemicals, Section 4. OECD Publishing, Paris. doi:10.1787/9789264229822-en

OECD (2018b). Test No. 442E: In Vitro Skin Sensitisation: In Vitro Skin Sensitisation assays addressing the Key Event on activation of dendritic cells on the Adverse Outcome Pathway for Skin Sensitisation. OECD Guidelines for the Testing of Chemicals, Section 4. OECD Publishing, Paris. doi:10.1787/9789264264359-en

Ostaszewski, K. and Rempala, G. A. (2000). Parametric and Nonparametric Bootstrap in Actuarial Practice. University of Louisville. https://bit.ly/2YzLGAP

Ramirez, T., Mehling, A., Kolle, S. N. et al. (2014). LuSens: A keratinocyte based ARE reporter gene assay for use in integrated testing strategies for skin sensitization hazard identification. Toxicol In Vitro 28, 1482-1497. doi:10.1016/j. tiv.2014.08.002

Ramirez, T., Stein, N., Aumann, A. et al. (2016). Intra- and inter-laboratory reproducibility and accuracy of the LuSens assay: A reporter gene-cell line to detect keratinocyte activation by skin sensitizers. Toxicol In Vitro 32, 278-286. doi:10.1016/j. tiv.2016.01.004

Reisinger, K., Hoffmann, S., Alépée, N. et al. (2015). Systematic evaluation of non-animal test methods for skin sensitisation safety assessment. Toxicol In Vitro 29, 259-270. doi:10.1016/j. tiv.2014.10.018

Russell, W. M. S. and Burch, R. L. (1959). Reduction - The problem of variance (Chapter 6). In The Principles of Humane Experimental Technique, Volume 238. London, UK: Methuen and Co., Ltd.

Sakaguchi, H., Ashikaga, T., Miyazawa, M. et al. (2006). Development of an in vitro skin sensitization test using human cell lines; human Cell Line Activation Test (h-CLAT) II. An inter-laboratory study of the h-CLAT. Toxicol In Vitro 20, 767773. doi:10.1016/j.tiv.2005.10.014

Sakaguchi, H., Ryan, C., Ovigne, J. M. et al. (2010). Predicting skin sensitization potential and inter-laboratory reproducibility of a human Cell Line Activation Test (h-CLAT) in the European Cosmetics Association (COLIPA) ring trials. Toxicol In Vitro 24, 1810-1820. doi:10.1016/j.tiv.2010.05.012

Sauer, U. G., Hill, E. H., Curren, R. D. et al. (2016). Local tolerance testing under REACH: Accepted non-animal methods are not on equal footing with animal tests. Altern Lab Anim 44, 281-299. doi:10.1177/026119291604400311

Sirota, N. P., Zhanataev, A. K., Kuznetsova, E. A. et al. (2014). Some causes of inter-laboratory variation in the results of comet assay. Mutat Res Genet Toxicol Environ Mutagen 770, 16-22. doi:10.1016/j.mrgentox.2014.05.003

Urbisch, D., Mehling, A., Guth, K. et al. (2015). Assessing skin sensitization hazard in mice and men using non-animal test methods. Regul Toxicol Pharmacol 71, 337-351. doi:10.1016/j.yrtph.2014.12.008

van der Schouw, Y. T., Verbeek, A. L., Ruijs, S. H. (1995). Guidelines for the assessment of new diagnostic tests. Invest Radiol 30, 334-340. doi:10.1097/00004424-199506000-00002

Wehrens, R., Putter, H., Buydens, L. M. C. (2000). The bootstrap: A tutorial. Chemometr Intell Lab Syst 54, 35-52. doi:10.1016/ S0169-7439(00)00102-7

Weil, C. S. and Scala, R. A. (1971). Study of intra- and interlaboratory variability in the results of rabbit eye and skin irritation tests. Toxicol Appl Pharmacol 19, 276-360. doi:10.1016/0041008X(71)90112-8

Worth, A. P. and Cronin, M. T. D. (2001a). The use of bootstrap resampling to assess the variability of Draize tissue scores. Altern Lab Anim 29, 557-573. doi:10.1177/026119290102900511

Worth, A. P. and Cronin, M. T. D. (2001b). The use of bootstrap resampling to assess the uncertainty of Cooper statistics. Altern Lab Anim 29, 447-459. doi:10.1177/026119290102900412

\section{Conflict of interest}

The authors declare they have no conflicts of interest.

\section{Acknowledgments}

We wish to thank Tzutzuy Fernandez Ramirez (BASF, Ludwigshafen, Germany) for providing us with the datasets used for LuSens prediction method. We also wish to thank Roman Liska (JRC, Ispra, Italy), Andrew Worth (JRC, Ispra, Italy), and Ekko C. Van Ierland (Wageningen UR, Wageningen, The Netherlands) for their valuable comments. 